\title{
Trajectory correction for lunar flyby transfers to libration point orbits using continuous thrust
}

\author{
Yi Qi ${ }^{1}$, Anton de Ruiter ${ }^{2}(\bowtie)$ \\ 1. School of Aerospace Engineering, Beijing Institute of Technology, Beijing 100081, China \\ 2. Department of Aerospace Engineering, Ryerson University, Toronto, ON M5B 2K3, Canada
}

\begin{abstract}
Trajectory corrections for lunar flyby transfers to Sun-Earth/Moon libration point orbits (LPOs) with continuous thrusts are investigated using an ephemeris model. The lunar flyby transfer has special geometrical and dynamical structures; therefore, its trajectory correction strategy is considerably different from that of previous studies and should be specifically designed. In this paper, we first propose a control strategy based on the backstepping technique with a dead-band scheme using an ephemeris model. The initial error caused by the launch time error is considered. Since the perturbed transfers significantly diverge from the reference transfers after the spacecraft passes by the Moon, we adopt two sets of control parameters in two portions before and after the lunar flyby, respectively. Subsequently, practical constraints owing to the navigation and propellant systems are introduced in the dynamical model of the trajectory correction. Using a prograde type 2 orbit as an example, numerical simulations show that our control strategy can efficiently address trajectory corrections for lunar flyby transfers with different practical constraints. In addition, we analyze the effects of the navigation intervals and dead-band scheme on trajectory corrections. Finally, trajectory corrections for different lunar flyby transfers are depicted and compared.
\end{abstract}

\section{KEYWORDS}

libration point orbit

trajectory correction

Earth-Moon system

continuous thrust

backstepping technique

Research Article

Received: 6 August 2020

Accepted: 25 October 2020

(C) The Author(s) 2020

\section{Introduction}

Because of the special dynamical properties of SunEarth/Moon libration point orbits (LPOs), many scientific and exploration missions to Sun-Earth/Moon LPOs have been implemented, such as ISEE-3 [1], WIND [2], and SOHO [3] for Sun-Earth/Moon $\mathrm{L}_{1}$ LPOs, MAP [4], GAIA [5], and CHANG'E-2 [6] for SunEarth/Moon $\mathrm{L}_{2}$ LPOs.

Many researchers focused on transfer problems to Sun-Earth/Moon LPOs. Gómez et al. applied the invariant manifolds associated with the LPOs to construct transfers from a low Earth orbit (LEO) to a SunEarth $\mathrm{L}_{1}$ halo orbit [7]. Based on the solution of the Lambert problem in the restricted three-body problem described by the Hill equations, Sukhanov and Prado proposed a design method for LEO-to-halo and halo-tohalo transfers [8]. Direct transfers to Sun-Earth LPOs increase the $\Delta v$ requirement owing to a tangential perigee velocity error and have the risk of being unable to perform the correction maneuver on day two. Therefore, Renk and Landgraf presented an indirect strategy to mitigate the criticality of the first correction maneuver of the transfer towards Sun-Earth LPOs by including an intermediate, highly elliptical parking orbit [9]. Using the CHANG'E-2 extension mission as an example, Peng et al. proposed an efficient GPU parallel computing technique to numerically search for transfers from a lunar orbit to the Sun-Earth $\mathrm{L}_{2}$ LPOs with different departing conditions using the patched elliptic restricted threebody problem model [10]. Their computationally efficient methodology obtained results almost identical to those of the ephemeris model and exhibited significant speedups. Qi et al. combined the technique of lunar flyby using the dynamical system approach, and they investigated 
lunar flyby transfers from an LEO to Sun-Earth/Moon LPOs [11]. The trajectory correction maneuver (TCM) problem is a significant problem associated with transfers to LPOs since perturbations and errors are inevitable during practical transfer missions. Farquhar et al. studied TCMs in the early transfer phase section of the ISEE3 mission [12]. Serban et al. investigated the TCM problem of the Genesis Discovery Mission using optimal control to compensate for launch vehicle errors, and they proposed two strategies to solve the TCM problem: the halo orbit insertion (HOI) and the manifold orbit insertion (MOI) techniques [13]. Gómez et al. presented a TCM strategy similar to the MOI technique for the TCM problem of the Genesis Mission, but they used a multiple shooting method instead of an optimal control procedure to address the TCM problem with a strong hyperbolic behavior of the orbits [14]. To correct the control errors and orbit determination errors, Wu et al. investigated the trajectory maneuvers before the Lissajous orbit insertion of the CHANG'E-2 mission from the Moon-circling orbit to Sun-Earth $\mathrm{L}_{2}[6]$. $\mathrm{Xu}$ and $\mathrm{Xu}$ applied the stochastic control theory for discrete linear stochastic systems to design a timing closed-loop TCM strategy during the transfer from an LEO to a Sun-Earth halo orbit in the circular restricted three-body problem (CRTBP) [15]. Salmani and Büskens proposed a real-time control method for the TCM of transfers to Sun-Earth $\mathrm{L}_{1}$ halo orbits in the Sun-Earth-Moon bicircular model [16]. They used an optimal control problem to prevent disturbances such as solar radiation and winds. Peng et al. researched the TCM problem of transferring a spacecraft with low thrust from an LEO to a Sun-Earth $\mathrm{L}_{2}$ halo orbit using a receding horizon control method [17].

Qi and de Ruiter investigated the TCM problem of lunar flyby transfers to Sun-Earth/Moon LPOs in the ephemeris model, and proposed several TCM strategies for lunar flyby transfers under practical constraints [18]. As stated in Ref. [18], the lunar flyby transfer is a high-yielding but high-risk design method because the perturbed lunar flyby transfers diverge significantly from the reference transfers after the spacecraft passes by the Moon. A two-impulse TCM must be executed before the lunar flyby for transfers from the Earth to LPOs to promptly restrain the divergence. In this paper, we focus on the trajectory correction for lunar flyby transfers to
Sun-Earth/Moon LPOs with continuous thrusts. Similar to the analysis in Ref. [18], according to the special geometrical structure of lunar flyby transfers, which are divided into two portions before and after lunar flyby, we postulate that a new design method must be developed to address the trajectory correction for lunar flyby transfers. Since the design of the reference transfers (defined as the transfer trajectory with no error) was adequately solved in the previous study [11], in this paper, we assume that the reference transfers are provided.

In this paper, trajectory corrections for lunar flyby transfers to Sun-Earth/Moon LPOs are investigated using the ephemeris model. In contrast to the previous study [18], the propellant system in this study was the continuous thrust rather than the impulsive thrust. We assume that the initial error is the launch time error, and we propose a control strategy based on the backstepping technique with a dead-band scheme. The backstepping technique has been widely applied in station-keeping [19, 20] and attitude tracking [21]. To the best of our knowledge, this is the first time that the backstepping technique is used in trajectory corrections for lunar flyby transfers. In contrast to the traditional applications of the backstepping technique, such as station-keeping and attitude tracking, since the perturbed transfers significantly diverge from the reference transfers after the spacecraft passes by the Moon, we should use two sets of control parameters in two portions before and after the lunar flyby. Compared with Ref. [18], more practical constraints in the trajectory correction will be considered in this paper, such as the dead-band scheme, navigation intervals, execution error, and limitation of the thrust engine. Therefore, the control strategy proposed herein is more realistic than that in the previous study. Through numerical simulations, we can analyze the effects of navigation intervals and dead-band schemes on trajectory corrections for different lunar flyby transfers.

The structure of this paper can be divided into five parts. In Section 2, we introduce the background, including the ephemeris model and lunar flyby transfers. In Section 3, we propose a control strategy for trajectory correction using continuous thrusts. In Section 4, practical constraints are introduced into the control strategy. In Section 5, numerical simulations are implemented and analyzed. Finally, the conclusions are presented in Section 6 . 


\section{Background}

\subsection{Ephemeris model}

Here, we use the J2000 Earth-centered inertial (ECI) frame to describe the motion of the spacecraft on lunar flyby transfers to LPOs. $\rho$ is the position vector of the spacecraft in the ECI frame. $\boldsymbol{P}_{i}$ and $i \in \Omega=\{$ Sun, Moon, Mercury, Venus, Mars, Jupiter, Saturn, Uranus, Neptune $\}$ represent the position vectors of the perturbing gravity bodies in the ECI frame. The position data of celestial bodies in the ECI frame can be obtained from the Jet Propulsion Laboratory (JPL) ephemeris DE430 [22]. The equation of motion of the spacecraft with propellant thrusts in the ECI frame can be expressed as

$$
\begin{aligned}
\ddot{\boldsymbol{\rho}}= & -G M_{\text {Earth }} \frac{\boldsymbol{\rho}}{\|\boldsymbol{\rho}\|^{3}} \\
& -\sum_{i \in \Omega}\left(G M_{i} \frac{\boldsymbol{\rho}-\boldsymbol{P}_{i}}{\left\|\boldsymbol{\rho}-\boldsymbol{P}_{i}\right\|^{3}}+G M_{i} \frac{\boldsymbol{P}_{i}}{\left\|\boldsymbol{P}_{i}\right\|^{3}}\right)+\boldsymbol{u}
\end{aligned}
$$

where $G$ is the gravitational constant, $M_{\text {Earth }}$ is the mass of the Earth, and $M_{i}$ is the corresponding mass of the celestial body in $\Omega . \boldsymbol{u}$ is the thrust acceleration provided by the propellant system.

For the continuous thrust implemented in the transfer orbit, we can obtain

$$
\begin{aligned}
\boldsymbol{u} & =\frac{\boldsymbol{T}}{m} \\
\dot{m} & =-\frac{T}{g_{0} I_{\mathrm{sp}}}
\end{aligned}
$$

where $\boldsymbol{T}=\left(T_{x}, T_{y}, T_{z}\right)$ represents the propellant thrust in the ECI frame; $T$ is the magnitude of the propellant thrust, i.e., $\|\boldsymbol{T}\| ; m$ is the spacecraft mass; $g_{0}$ is the acceleration due to gravity at sea level, and is equal to $9.80665 \mathrm{~m} / \mathrm{s}^{2}$; and $I_{\mathrm{sp}}$ is the specific impulse of the engine. The data of the spacecraft and thrust engine are listed in Table 1. A BHT-200 Busek Hall effect thruster can provide $13 \mathrm{mN}$ of thrust at $200 \mathrm{~W}$ power and a specific impulse of 1375 s (http://www.busek.com/index_htm files/70000700A\%20BHT-200.pdf). Hence, if we deploy two thrusters such as the BHT-200 thrusters in the spacecraft, the performance requirement for thrusters

Table 1 Spacecraft properties

\begin{tabular}{lcc}
\hline Name & Symbol & Value \\
\hline Initial spacecraft mass & $m_{0}$ & $500 \mathrm{~kg}$ \\
Maximum thrust & $T_{\max }$ & $26 \mathrm{mN}$ \\
Engine number & $n$ & 2 \\
Specific impulse & $I_{\mathrm{sp}}$ & $1000 \mathrm{~s}$ \\
\hline
\end{tabular}

listed in Table 1 can be realized.

\subsection{Lunar flyby transfers to libration point orbits}

In this paper, lunar flyby transfers from an LEO at an altitude of $200 \mathrm{~km}$ to Sun-Earth/Moon LPOs are analyzed. Based on Ref. [11], eight types of lunar flyby transfers exist: prograde types 1-4 transfers and retrograde types 1-4 transfers. The eight types of lunar flyby transfers exhibit no apparent differences in terms of fuel consumption. However, for $\mathrm{L}_{1}$ target LPOs, prograde and retrograde type 2 transfers require shorter transfer durations than those of type 1 transfers; for $\mathrm{L}_{2}$ target LPOs, prograde and retrograde type 4 transfers require shorter transfer durations than those of type 3 transfers [11]. Hence, prograde and retrograde types 2 and 4 transfers are preferable in LPOs missions. Figure 1 shows four types of lunar flyby transfers to Sun-Earth/Moon LPOs: (a) prograde type 2 transfer to an $\mathrm{L}_{1} \mathrm{LPO}$, (b) retrograde type 2 transfer to an $\mathrm{L}_{1}$ LPO, (c) prograde type 4 transfer to an $\mathrm{L}_{2} \mathrm{LPO}$, and (d) retrograde type 4 transfer to an $\mathrm{L}_{2}$ LPO, where the black and red lines denote the transfer orbits and target LPOs, respectively. These four target LPOs are actually Lissajous orbits, and we assume that they remain unchanged in the trajectory correction problem. The transfer orbits in Fig. 1 are described in the dimensionless Sun-Earth/Moon rotating frame and calculated in the ephemeris model. The length unit of the dimensionless Sun-Earth/Moon rotating frame is the instantaneous distance between the Sun and the Earth-Moon barycenter (EMB). At the initials of lunar flyby transfers, a tangential maneuver $\Delta v_{\text {in }}$ is implemented to escape the initial LEO at an altitude of $200 \mathrm{~km}$. Subsequently, at the terminals of the lunar flyby transfers, i.e., insertion points (blue points in Fig. 1), a tangential impulsive maneuver $\Delta v_{\text {end }}$ is implemented to insert the spacecraft into target LPOs. Table 2 lists the data of the four transfers in Fig. 1, where $i_{\text {leo }}$ denotes the inclination of the initial LEO in the ECI frame.

Although lunar flyby transfers to LPOs have advantages in terms of fuel consumption and flight time [11], they are more delicate and unstable than traditional transfers without lunar flybys [18]. The design aim of the trajectory correction in this paper is to enable the spacecraft to be inserted into the target LPO. Hence, trajectory correction with continuous thrust is executed 


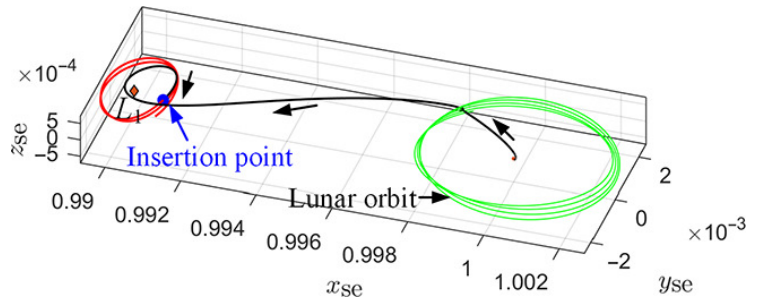

(a) Prograde type 2 transfer to an $\mathrm{L}_{1} \mathrm{LPO}$

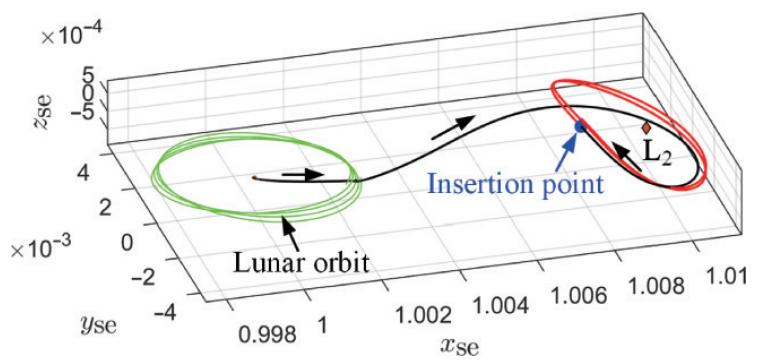

(c) Prograde type 4 transfer to an $\mathrm{L}_{2} \mathrm{LPO}$

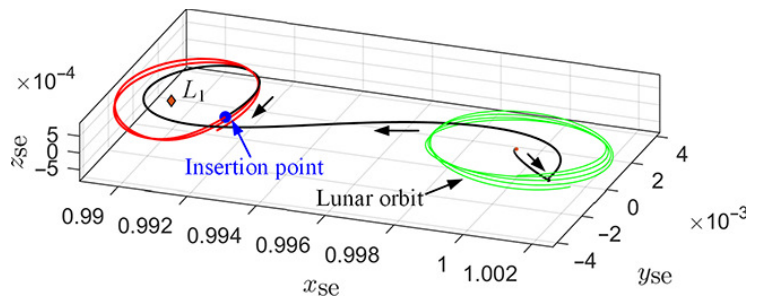

(b) Retrograde type 2 transfer to an $\mathrm{L}_{1} \mathrm{LPO}$

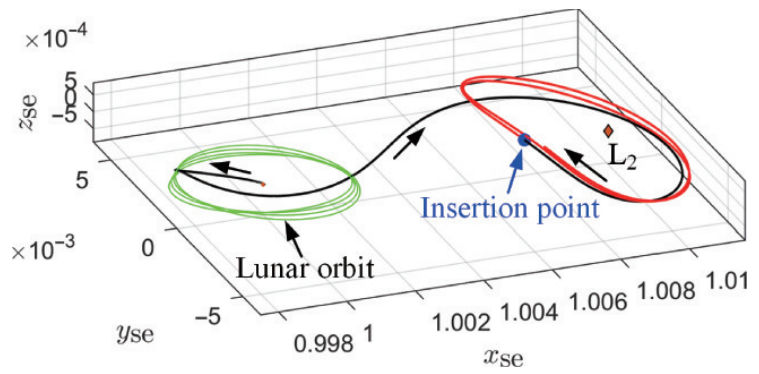

(d) Retrograde type 4 transfer to an $\mathrm{L}_{2}$ LPO

Fig. 1 Four types of lunar flyby transfers in the dimensionless Sun-Earth/Moon rotating frame; the black and red lines denote transfer orbits and target LPOs, respectively.

Table 2 Data of the four lunar flyby transfers in Fig. 1

\begin{tabular}{ccccccc}
\hline Orbit & Start time (UTC) & Perilune time (UTC) & Insertion time (UTC) & $i_{\text {leo }}(\operatorname{deg})$ & $\Delta v_{\text {in }}(\mathrm{m} / \mathrm{s})$ & $\Delta v_{\text {end }}(\mathrm{m} / \mathrm{s})$ \\
\hline a & $2020-08-11 ~ 14: 48: 16$ & $2020-08-1522: 25: 25$ & $2021-01-1319: 30: 07$ & 24.1822 & 3128 \\
b & $2020-07-2510: 24: 30$ & $2020-07-3015: 59: 30$ & $2021-01-0201: 01: 19$ & 24.1763 & 3133 \\
c & $2020-07-2902: 36: 13$ & $2020-08-0208: 27: 33$ & $2020-12-3108: 54: 52$ & 24.0894 & 3129 & 3.0691 \\
d & $2020-08-1010: 43: 24$ & $2020-08-1523: 01: 51$ & $2021-01-2104: 30: 06$ & 24.1849 & 3134 & 5.6639 \\
\hline
\end{tabular}

during the transfer. In this paper, we select the above four lunar flyby transfers as reference orbits of the trajectory correction. In addition, we note that in Table 2, the insertion maneuvers of the reference orbits $\Delta v_{\text {end }}$ are considerably small; hence, a portion of the target LPO orbit after the insertion point is added into the reference orbit, and we implement continuous thrusts to achieve the insertion instead of the small impulsive maneuvers. In other words, the reference orbit considered in this paper includes the lunar flyby transfer orbit and a portion of the target LPO.

In practice, various types of errors occur at the initial point introduced by the inaccuracies of the launch vehicle, such as the velocity error $[13,18,20]$ and position error [20]. In this paper, we propose an initial error caused by the launch time error $t_{\text {err }}$ in the initial LEO at an altitude of $200 \mathrm{~km}$. Thus, the launch time error, regardless of the delay or advance, in the initial LEO results in velocity and position errors.

\section{Control strategy}

In this section, we propose a control strategy for trajectory correction with continuous thrusts in the ephemeris model. The control strategy is based on the backstepping technique, which has been widely applied in station-keeping [19, 20] and attitude tracking [21].

Based on Eq. (1), the controlled equation of motion of the spacecraft in the ephemeris model can be rewritten as

$$
\dot{\boldsymbol{x}}=\boldsymbol{f}(t, \boldsymbol{x})+\boldsymbol{B} \boldsymbol{u}(t)
$$

where $\boldsymbol{x}=[\boldsymbol{\rho}, \boldsymbol{v}]$ is the phase state, including the position and velocity state, of the spacecraft in the ECI frame; $\boldsymbol{f}=[\boldsymbol{v}, \boldsymbol{a}]$, where $\boldsymbol{a}$ is the gravity acceleration, i.e., the first and second terms of the right-hand side in Eq. (1); and $\boldsymbol{B}=\left[\begin{array}{lll}\boldsymbol{O}_{3 \times 3} & \boldsymbol{I}_{3 \times 3}\end{array}\right]^{\mathrm{T}}$, where $\boldsymbol{O}_{3 \times 3}$ and $\boldsymbol{I}_{3 \times 3}$ are $3 \times 3$ zero matrix and identity matrix, respectively. The state deviation with respect to the reference orbit is $\Delta \boldsymbol{x}=$ $\boldsymbol{x}-\boldsymbol{x}_{\mathrm{R}}$, where $\boldsymbol{x}_{\mathrm{R}}=\left[\boldsymbol{\rho}_{\mathrm{R}}, \boldsymbol{v}_{\mathrm{R}}\right]$ is the state of the reference orbit; therefore, we can obtain $\dot{\boldsymbol{x}}_{\mathrm{R}}=\boldsymbol{f}\left(t, \boldsymbol{x}_{\mathrm{R}}\right)$. Thus, we 
can derive the linear dynamical equation of the state deviation as Eq. (4):

$$
\begin{aligned}
\Delta \dot{\boldsymbol{x}} & =\boldsymbol{A}\left(t, \boldsymbol{x}_{\mathrm{R}}\right) \Delta \boldsymbol{x}+\boldsymbol{B} \boldsymbol{u}(t) \\
& =\left(\begin{array}{cc}
\boldsymbol{O}_{3 \times 3} & \boldsymbol{I}_{3 \times 3} \\
\boldsymbol{A}_{21}(t, \boldsymbol{\rho}) & \boldsymbol{O}_{3 \times 3}
\end{array}\right)\left(\begin{array}{c}
\Delta \boldsymbol{\rho} \\
\Delta \boldsymbol{v}
\end{array}\right)+\boldsymbol{B} \boldsymbol{u}(t)
\end{aligned}
$$

where $\boldsymbol{A}$ is the Jacobi matrix and $\boldsymbol{A}_{21}(t, \boldsymbol{\rho})=\partial \boldsymbol{a} / \partial \boldsymbol{\rho}$.

Since the phase state of the reference orbit $\boldsymbol{x}_{\mathrm{R}}(t)$ is known, $\boldsymbol{A}\left(t, \boldsymbol{x}_{N}\right)=\boldsymbol{A}(t)$ only depends on time. The classical optimal linear quadratic regulator (LQR) control technique to minimize the quadratic cost function:

$$
J=\int_{t_{0}}^{t_{\mathrm{f}}}\left[\Delta \boldsymbol{x}^{\mathrm{T}}(t) \boldsymbol{Q} \Delta \boldsymbol{x}(t)+\boldsymbol{u}^{\mathrm{T}}(t) \boldsymbol{R} \boldsymbol{u}(t)\right] \mathrm{d} t
$$

requires a solution to the time-varying Riccati equation. To avoid repetitively solving the Riccati equation, we can use the backstepping technique proposed by Nazari et al. to reduce the original system into a time-invariant subsystem for which it is easier to obtain the control law stabilizing this subsystem [19]. Based on the result of the subsystem, we can derive the control law that stabilizes the original system through backstepping.

According to Refs. [19, 23], the backstepping transformation is expressed as

$$
\begin{aligned}
& \boldsymbol{z}_{1}=\Delta \boldsymbol{x}_{1} \\
& \boldsymbol{z}_{2}=\Delta \boldsymbol{x}_{2}-\boldsymbol{S} \Delta \boldsymbol{x}_{1}
\end{aligned}
$$

where $\Delta \boldsymbol{x}_{1}=\boldsymbol{\rho}-\boldsymbol{\rho}_{\mathrm{R}}$ and $\Delta \boldsymbol{x}_{2}=\boldsymbol{v}-\boldsymbol{v}_{\mathrm{R}}$ are the position and velocity deviations with respect to the reference orbit, respectively, and $\boldsymbol{S}$ is a constant negative definite matrix.

According to Eq. (4), we obtain

$$
\begin{aligned}
& \Delta \dot{\boldsymbol{x}}_{1}=\Delta \boldsymbol{x}_{2} \\
& \Delta \dot{\boldsymbol{x}}_{2}=\boldsymbol{A}_{21}(t) \Delta \boldsymbol{x}_{1}+\boldsymbol{u}
\end{aligned}
$$

Hence, taking the derivative of Eq. (5) with respect to $t$, we obtain

$$
\begin{aligned}
& \dot{\boldsymbol{z}}_{1}=\boldsymbol{S} \boldsymbol{z}_{1}+\boldsymbol{z}_{2} \\
& \dot{\boldsymbol{z}}_{2}=\left(\boldsymbol{A}_{21}(t)-\boldsymbol{S}^{2}\right) \boldsymbol{z}_{1}-\boldsymbol{S} \boldsymbol{z}_{2}+\boldsymbol{u}
\end{aligned}
$$

Let $\boldsymbol{u}=-\left(\boldsymbol{A}_{21}-\boldsymbol{S}^{2}\right) \boldsymbol{z}_{1}+\boldsymbol{u}_{1}$; thus, we can obtain a time-invariant subsystem:

$$
\dot{\boldsymbol{z}}_{2}=-\boldsymbol{S} \boldsymbol{z}_{2}+\boldsymbol{u}_{1}(t)
$$

For the time-invariant subsystem (8), we assume that $\boldsymbol{u}_{1}(t)=-\boldsymbol{k} \boldsymbol{z}_{2}(t)$, where $\boldsymbol{k}$ should satisfy the minimization of the quadratic cost function:

$$
J=\int_{0}^{\infty}\left[\boldsymbol{z}_{2}^{\mathrm{T}}(t) \boldsymbol{Q} \boldsymbol{z}_{2}(t)+\boldsymbol{u}_{1}^{\mathrm{T}}(t) \boldsymbol{R} \boldsymbol{u}_{1}(t)\right] \mathrm{d} t
$$

where $\boldsymbol{Q}$ and $\boldsymbol{R}$ are constant weighting matrices, $\boldsymbol{Q} \in$ $\mathbb{R}^{3 \times 3}$ is a positive semi-definite matrix, and $\boldsymbol{R} \in \mathbb{R}^{3 \times 3}$ is a positive definite matrix. To calculate $\boldsymbol{k}$, we must solve an algebraic Riccati equation (ARE) for the infinite-horizon problem:

$$
\boldsymbol{S}^{\mathrm{T}} \boldsymbol{P}+\boldsymbol{P} \boldsymbol{S}-\boldsymbol{Q}+\boldsymbol{P} \boldsymbol{R}^{-1} \boldsymbol{P}=\boldsymbol{O}_{3 \times 3}
$$

Subsequently, the control gain of subsystem (8), $\boldsymbol{k}$, can be obtained using $\boldsymbol{k}=\boldsymbol{R}^{-1} \boldsymbol{P}$. Therefore, in the numerical computation of $\boldsymbol{k}$, we only require to solve an ARE once.

For the original system (3), the control input can be expressed as

$$
\begin{aligned}
\boldsymbol{T} / m & =\boldsymbol{u}=-\left(\boldsymbol{A}_{21}-\boldsymbol{S}^{2}\right) \boldsymbol{z}_{1}-\boldsymbol{k} \boldsymbol{z}_{2}(t) \\
& =-\left(\boldsymbol{A}_{21}-\boldsymbol{S}^{2}+\boldsymbol{k} \boldsymbol{S}\right) \Delta \boldsymbol{x}_{1}-\boldsymbol{k} \Delta \boldsymbol{x}_{2}=-\boldsymbol{K} \Delta \boldsymbol{x}
\end{aligned}
$$

where $\boldsymbol{A}_{21}$ can be obtained from the reference orbit, i.e., $\boldsymbol{A}_{21}(t, \boldsymbol{\rho})=\partial \boldsymbol{a}_{\mathrm{R}} / \partial \boldsymbol{\rho}_{\mathrm{R}}$.

Based on the above analysis, the performance of the backstepping technique depends on the selection of parameters $\boldsymbol{S}, \boldsymbol{Q}$, and $\boldsymbol{R}$. When the values of these three parameters are known, the control strategy is specified. In the station-keeping problem $[19,20], \boldsymbol{S}, \boldsymbol{Q}$, and $\boldsymbol{R}$ are fixed during the entire mission. However, lunar flyby transfers are considerably different from LPOs in the station-keeping problem. The most noticeable difference is that the geometrical structure and orbital environment of lunar flyby transfers are divided into two portions before and after the lunar flyby. Based on Ref. [24], the prior-lunar flyby can be approximately analyzed in the Earth-Moon CRTBP, while the post-lunar flyby can be approximately studied in the Sun-Earth CRTBP. In Ref. [18], an error analysis before and after lunar flyby indicated that, compared with the transfer without a lunar flyby, the initial error of the lunar flyby transfer is amplified by the lunar flyby. Therefore, we postulate that two sets of the parameters $\boldsymbol{S}, \boldsymbol{Q}$, and $\boldsymbol{R}$ should be adopted in two portions before and after the lunar flyby.

First, we estimate the magnitude of $\boldsymbol{S}$, i.e., $\|\boldsymbol{S}\|$. As mentioned earlier, we use the launch time error $\left(t_{\text {err }}\right)$ to create initial velocity and position errors, and when $t_{\text {err }}$ is known, the perturbed orbit is specified and can be obtained using numerical integration. Using the prograde type 2 transfer in Fig. 1(a) as an example, we assume that the launch time on the initial LEO of altitude $200 \mathrm{~km}$ is postponed for $10 \mathrm{~s}$, i.e., $t_{\mathrm{err}}=10 \mathrm{~s}$. In addition, owing to the limitation of navigation technology, the trajectory correction cannot be performed until a minimum of 12 hours after launch. Numerical computation indicates that 
when the continuous thrust begins to operate 12 hours after the launch, the position and velocity errors from the reference orbit here are approximately $1545 \mathrm{~km}$ and $24 \mathrm{~m} / \mathrm{s}$, respectively. Hence, $\left\|\Delta \boldsymbol{x}_{2}\right\|$ is approximately $0.024 \mathrm{~km} / \mathrm{s}$, and $\left\|\Delta \boldsymbol{x}_{1}\right\|$ is approximately $1545 \mathrm{~km}$. Since $\boldsymbol{z}_{1}$ and $\boldsymbol{z}_{2}$ in Eq. (5) are the equivalent parameters of $\Delta \boldsymbol{x}_{1}$ and $\Delta \boldsymbol{x}_{2}$, respectively, based on the second equation of Eq. (5), we estimate that $\|\boldsymbol{S}\|$ should be smaller than $0.024 / 1545 \approx 1.5 \times 10^{-5}$. The purpose of the backstepping control is to drive $z_{2}$ to zero asymptotically. Based on Eq. (5), if we set $z_{2}=0, \Delta \dot{x}_{1}=\Delta x_{2}=S \Delta x_{1}$, which has the solution $\Delta x_{1}(t)=\exp (S t) \Delta x_{1}(0)$. Hence, the magnitude of $S$ can also determine the rate of convergence of the position error.

Second, we discuss the relationship between $Q$ and $\boldsymbol{R}$. In the cost function $(J)$ in Eq. (9), the terms $\boldsymbol{z}_{2}^{\mathrm{T}} \boldsymbol{Q} \boldsymbol{z}_{2}$ and $\boldsymbol{u}_{1}^{\mathrm{T}} \boldsymbol{R} \boldsymbol{u}_{1}$ represent the performance indices of the trajectory correction and fuel consumption, respectively. For a fixed $\|\boldsymbol{R}\|$, a larger $\|\boldsymbol{Q}\|$ can increase the convergence rate of the perturbed orbit to the reference orbit, but the thrust acceleration $(u)$ will also increase; otherwise, the decrease in $\|\boldsymbol{Q}\|$ can retard the convergence rate of the perturbed orbit and reduce the thrust acceleration. As previously discussed, $\left\|\boldsymbol{z}_{2}\right\|$ is the same as $\left\|\Delta \boldsymbol{x}_{2}\right\|$, i.e., approximately $24 \mathrm{~m} / \mathrm{s}$. For the thrust engine in Table 1, the maximum magnitude of the thrust acceleration $\|\boldsymbol{u}\|$ is approximately $T_{\max } / m_{0}=$ $5.2 \times 10^{-5} \mathrm{~m} / \mathrm{s}^{2}$. We assume that the terms $\boldsymbol{z}_{2}^{\mathrm{T}} \boldsymbol{Q} \boldsymbol{z}_{2}$ and $\boldsymbol{u}_{1}^{\mathrm{T}} \boldsymbol{R} \boldsymbol{u}_{1}$ have similar magnitudes to balance the trajectory correction and thrust acceleration. Subsequently, we can estimate that $\|\boldsymbol{Q}\| \approx 4.5 \times 10^{-12}\|\boldsymbol{R}\|$. However because the perturbed transfers significantly diverge from the reference transfers after the spacecraft passes by the Moon [18], the convergence rate of the perturbed orbit before the lunar flyby, rather than the decrease in thrust acceleration, should be prioritized.

As stated earlier, the magnitudes of $\boldsymbol{S}, \boldsymbol{Q}$, and $\boldsymbol{R}$ can affect the convergence rate of the perturbed orbit. For convenience, we fix $\boldsymbol{S}$ and $\boldsymbol{R}$ at constant values and use the magnitude of $\boldsymbol{Q}$ to change the rate of convergence for portions before and after the lunar flyby. Based on the above discussion, $\boldsymbol{S}$ is a negative definite matrix and $\|\boldsymbol{S}\|$ should be smaller than $1.5 \times 10^{-5}$; therefore, for the entire transfer, we set $\boldsymbol{S}=-4 \times 10^{-6} \boldsymbol{I}_{3 \times 3} . \boldsymbol{R}$ is a positive definite matrix, and numerical computation indicates that $\boldsymbol{R}=10^{6} \boldsymbol{I}_{3 \times 3}$ is feasible for the entire transfer. For the portion after the lunar flyby, we use the above estimation equation of $\boldsymbol{Q}$ and $\boldsymbol{R}$ and set $\boldsymbol{Q}=10^{-5} \boldsymbol{I}_{3 \times 3}$. For the portion before the lunar flyby, we adopt a larger $\boldsymbol{Q}$ compared with the estimation equation to increase the convergence rate of the perturbed orbit; therefore, we set $\boldsymbol{Q}=0.6 \boldsymbol{I}_{3 \times 3}$. To distinguish these two values of $\boldsymbol{Q}$ before and after the lunar flyby, we denote them as $\boldsymbol{Q}_{1}$ and $\boldsymbol{Q}_{2}$, respectively. For the application scope of $\boldsymbol{Q}_{1}$, we postulate that it should encompass the portion before the lunar flyby and the process of the lunar flyby. Based on the geometrical structure of the lunar flyby transfers (Fig. 1), the region in which the distance of the spacecraft to the EMB is smaller than $1.1 a_{\mathrm{m}}\left(1+e_{\mathrm{m}}\right)$ can seemingly satisfy the above requirement, where $a_{\mathrm{m}}$ denotes the semi-major axis of the lunar orbit (approximately $3.8476 \times 10^{5} \mathrm{~km}$ ) and $e_{\mathrm{m}}$ is the eccentricity of the lunar orbit (approximately 0.0549). Outside this region, $\boldsymbol{Q}_{2}$ is appropriate.

For instance, we use an idealistic simulation to compare the effects of $\boldsymbol{Q}_{1}$ on the trajectory correction of the prograde type 2 transfer in Fig. 1(a). The idealistic simulation requires that practical errors, such as navigation or execution errors, and the limitation of the thrust engine, such as the maximum thruster $T_{\max }$, are not considered. If $t_{\mathrm{err}}=10 \mathrm{~s}, \boldsymbol{S}=-4 \times 10^{-6} \boldsymbol{I}_{3 \times 3}$, $\boldsymbol{R}=10^{6} \boldsymbol{I}_{3 \times 3}$, and $\boldsymbol{Q}_{2}=10^{-5} \boldsymbol{I}_{3 \times 3}$, Fig. 2 shows performances of two types of trajectory corrections for $\boldsymbol{Q}_{1}=0.6 \boldsymbol{I}_{3 \times 3}$ and $10^{-5} \boldsymbol{I}_{3 \times 3}$, where $d$ and $\Delta v$ denote the distance and velocity difference of the perturbed orbit from the reference orbit, respectively. In Fig. 2, the
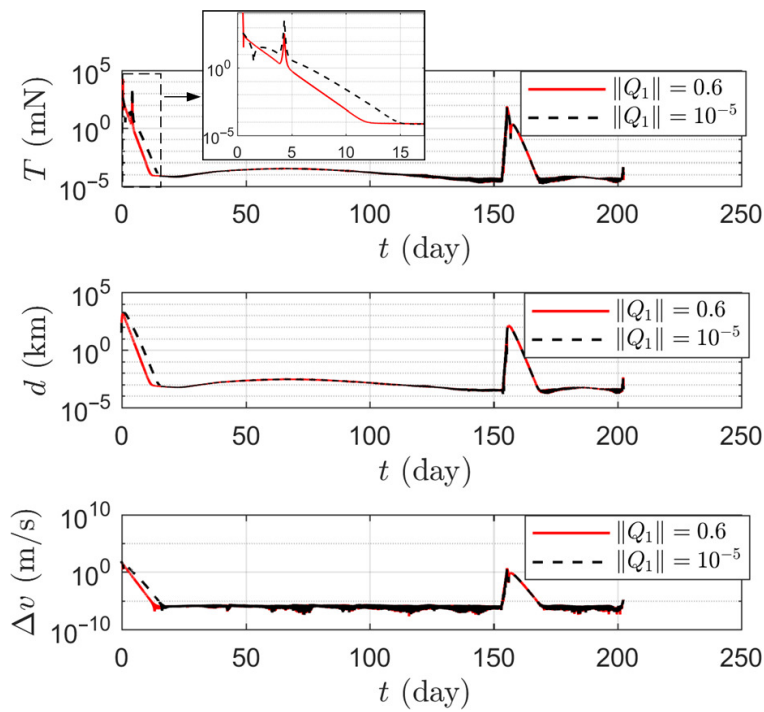

Fig. 2 Performances of two types of trajectory corrections for $\boldsymbol{Q}_{1}=0.6 \boldsymbol{I}_{3 \times 3}$ and $10^{-5} \boldsymbol{I}_{3 \times 3}$. 
large divergence of the perturbed and reference orbits in the beginning is caused by the initial launch time error; the other large divergence of the perturbed and reference orbits at $t=153$ days is caused by the insertion maneuver in the reference orbit. As the figure shows, the trajectory correction with $\|\boldsymbol{Q}\|=0.6$ requires a larger $T$ at the beginning of the trajectory correction, but after that, its thrust $T, d$, and $\Delta v$ are all smaller than those for $\|\boldsymbol{Q}\|=10^{-5}$. This result confirms our earlier inference that a larger $\|\boldsymbol{Q}\|$ can increase the convergence rate of the perturbed orbit to the reference orbit, but the thrust acceleration $(u)$ will also increase. In addition, numerical computation indicates that the propellant mass losses, i.e., $m_{\text {loss }}$, for trajectory corrections with $\left\|\boldsymbol{Q}_{1}\right\|=0.6$ and $10^{-5} \boldsymbol{I}_{3 \times 3}$ are 4.1691 and $4.7528 \mathrm{~kg}$, respectively. Therefore, for the total fuel consumption, using a larger $\left\|\boldsymbol{Q}_{1}\right\|$ to promptly reduce the position and velocity errors before the lunar flyby is more reasonable.

In addition, as stated in Ref. [20], a dead-band scheme can avoid thrusters operating continuously for long-term maneuvers. Figure 3 shows the schematic of a deadband scheme. It assumes that thrusters shut down when the distance of the spacecraft from the reference orbit decreases to a lower boundary (which is denoted by $d_{\text {low }}$ ) from a farther position; subsequently, thrusters start up when the distance from the reference orbit increases to an upper boundary (which is denoted by $d_{\text {up }}$ ) from a closer position. The interval times between $t_{1}$ and $t_{2}$ in Fig. 3 is the idle time of the thrusters. In this paper, unless explicitly stated, $d_{\text {low }}$ and $d_{\text {up }}$ are set to 5 and $20 \mathrm{~km}$, respectively.

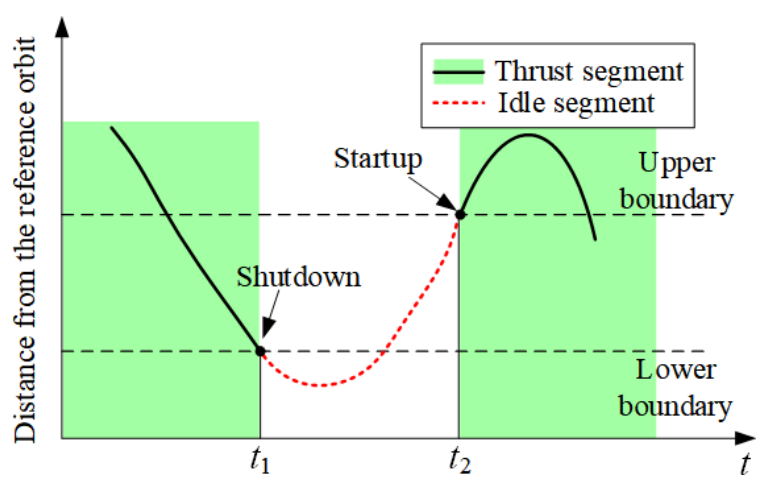

Fig. 3 Schematic of a dead-band scheme.

\section{Practical constraints}

In this section, practical constraints during trajectory correction are introduced. Two sources of practical constraints exist: the navigation and propellant systems.

In the transfer mission to a Sun-Earth/Moon LPO, the navigation system cannot provide updates in real time and requires a navigation interval. Let $t_{i}$ and $\Delta T_{n i}$ denote the navigation epoch and navigation interval time, respectively. Based on Eq. (4), in the navigation interval $\left[t_{i}, t_{i}+\Delta T_{n i}\right]$, the state error can be obtained using a forward numerical integration of the linear dynamical system as Eq. (12):

$$
\begin{aligned}
\Delta \dot{\boldsymbol{x}} & =\boldsymbol{A}\left(t, \boldsymbol{x}_{\mathrm{R}}\right) \Delta \boldsymbol{x}+\frac{\boldsymbol{B} \boldsymbol{T}(t)}{m} \\
\dot{m} & =-\frac{T}{g_{0} I_{\mathrm{sp}}} \\
\Delta \boldsymbol{x}\left(t_{i}\right) & =\Delta \boldsymbol{x}_{n i}
\end{aligned}
$$

where $\Delta \boldsymbol{x}_{n i}$ is the state error with respect to the reference orbit obtained from the navigation system at $t_{i}$. In the navigation interval $\left[t_{i}, t_{i}+\Delta T_{n i}\right], \Delta \boldsymbol{x}$ obtained from the linear dynamical system (12) is the pseudo state error rather than the actual state error. In Eq. (12), $\boldsymbol{A}\left(t, \boldsymbol{x}_{\mathrm{R}}\right)=\boldsymbol{A}(t)$ only depends on time and can be obtained a priori; thus, the computational cost of the numerical integration of the linear system (12) is lower than that of the actual ephemeris model and is more computationally implementable on a flight processor.

The navigation error is denoted by $\varepsilon_{n}=\left[\begin{array}{lll}\varepsilon_{X} & \varepsilon_{Y} & \varepsilon_{Z} \\ \varepsilon_{\dot{X}}\end{array}\right.$ $\left.\varepsilon_{\dot{Y}} \varepsilon_{\dot{Z}}\right]^{\mathrm{T}}$. Thus, the actual state error obtained from the navigation system at time $t_{i}$ can be expressed as

$$
\Delta \boldsymbol{x}_{n i}=\Delta \boldsymbol{x}_{\text {real }}\left(t_{i}\right)+\boldsymbol{\varepsilon}_{n}
$$

where $\Delta \boldsymbol{x}_{\text {real }}\left(t_{i}\right)$ is the actual state error without errors.

Similar to the analysis in $\boldsymbol{Q}$, we consider that using two values of the navigation interval time $\left(\Delta T_{n i}\right)$ before and after the lunar flyby is necessary because the lunar flyby can enlarge the divergence of the perturbed transfer from the reference transfer [18]. Thus, a short $\Delta T_{n i}$ before the lunar flyby can increase the accuracy of the pseudo state error in the portion before the lunar flyby. Let $\Delta T_{n i 1}$ and $\Delta T_{n i 2}$ represent the values of $\Delta T_{n i}$ before and after the lunar flyby, respectively, with $\Delta T_{n i 1}<\Delta T_{n i 2}$. For the application scope of $\Delta T_{n i 1}$, we assume that it is identical to that of $\boldsymbol{Q}_{1}$, i.e., the distance of the spacecraft to the EMB should be smaller than $1.1 a_{\mathrm{m}}\left(1+e_{\mathrm{m}}\right)$.

Based on Eq. (11) and the pseudo state error $(\Delta \boldsymbol{x})$, the idealistic thrust in the navigation interval $\left[t_{i}, t_{i}+\Delta T_{n i}\right]$ can be expressed as

$$
\overline{\boldsymbol{T}}(t)=-m \boldsymbol{K}\left(t, \boldsymbol{x}_{\mathrm{R}}\right) \Delta \boldsymbol{x}
$$


If the engine limitation is considered, $\overline{\boldsymbol{T}}(t)$ can be rewritten as

$$
\boldsymbol{T}^{*}(t)= \begin{cases}\mathbf{0}, & \bar{T}<T_{\min } \\ \frac{T_{\max }}{\bar{T}}, & \bar{T}>T_{\max } \\ \overline{\boldsymbol{T}}, & \bar{T} \in\left[T_{\min }, T_{\max }\right]\end{cases}
$$

where $T_{\min }$ and $T_{\max }$ are the minimum and maximum magnitudes of the thrust, respectively.

Let $\varepsilon_{T_{i}},(i=X, Y, Z)$ denote the execution error. Thus, the control input executed in Eq. (12) is

$$
\boldsymbol{T}(t)= \begin{cases}\mathbf{0}, & T^{*}<T_{\min } \\ \boldsymbol{E}_{\mathrm{m}} \boldsymbol{T}^{*}, & T^{*} \geqslant T_{\min }\end{cases}
$$

where $\boldsymbol{E}_{\mathrm{m}}=\operatorname{diag}\left[1+\varepsilon_{T_{X}}, 1+\varepsilon_{T_{Y}}, 1+\varepsilon_{T_{Z}}\right]$.

Using Eqs. (12)-(16), a control strategy with practical constraints in the navigation interval $\left[t_{i}, t_{i}+\Delta T_{n i}\right]$ is established. We assume that both the navigation and execution errors are white noise processes and have standard normal distributions with zero means. The parameters of practical constraints are listed in Table 3, for which the parameters of the navigation system were obtained from the navigation uncertainties of the ARTEMIS mission [25]. $T_{\max }$ is obtained from Table 1 , and $T_{\min }$ is equal to $1 \%$ of the maximum thrust of a single thruster. In addition, we assume that the initial launch time error is known in the trajectory correction.

Table 3 Practical constraints

\begin{tabular}{lc}
\hline Parameter & Value \\
\hline Navigation errors & \\
$\sigma_{X_{n}, Y_{n}, Z_{n}}$ & $1 \mathrm{~km}$ \\
$\sigma_{\dot{X}_{n}, \dot{Y}_{n}, \dot{Z}_{n}}$ & $1 \mathrm{~cm} / \mathrm{s}$ \\
\hline Execution errors & \\
$\sigma_{T_{X}, T_{Y}, T_{Z}}$ & $2 \%$ \\
\hline Engine limitation & \\
$T_{\max }$ & $26 \mathrm{mN}$ \\
$T_{\min }$ & $0.13 \mathrm{mN}$ \\
\hline
\end{tabular}

\section{$5 \quad$ Numerical simulations}

\subsection{Effects of navigation intervals}

In this subsection, we investigate the effects of navigation intervals on the trajectory correction of the lunar flyby transfer using Monte Carlo simulations. Specifically, the prograde type 2 transfer shown in Fig. 1(a) is used as the reference orbit in numerical simulations of this subsection, and the dead-band scheme and practical constraints are all considered. Figure 4 shows the statistical results of
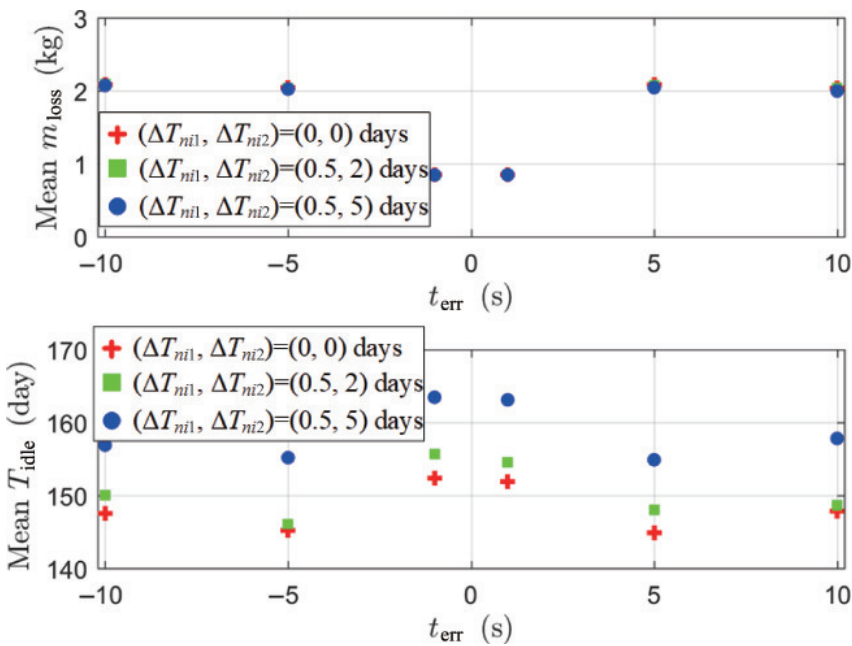

Fig. 4 Statistical results of trajectory corrections with different navigation intervals and launch time errors $t_{\mathrm{err}}$.

trajectory corrections with different navigation intervals, where $T_{\text {idle }}$ represents the total idle time. For the control strategy with a dead-band scheme, the total idle time $T_{\text {idle }}$ includes two parts: the idle time of the dead-band scheme and the idle time of $\bar{T}<T_{\min }$, but for the control strategy without a dead-band scheme, only the latter exists. For each simulation with particular values of $t_{\mathrm{err}}$ and $\left(\Delta T_{n i 1}, \Delta T_{n i 2}\right), 100$ sample points with practical constraints were selected. As mentioned earlier, the navigation and execution errors are white noise processes and have standard normal distributions with zero means. As Fig. 4 shows, for the particular launch time error $t_{\text {err }}$, trajectory corrections with different navigation intervals had almost similar mean fuel consumptions or mass losses $m_{\text {loss }}$; therefore, the marks for $(0,0)$ and $(0.5,2)$ days appeared covered by the marks for $(0.5,5)$ days. However, the trajectory correction with a longer navigation interval had a longer $T_{\text {idle }}$. In addition, the figure show that trajectory corrections with $\left|t_{\mathrm{err}}\right|=1 \mathrm{~s}$ had a lower fuel consumption or $m_{\text {loss }}$ but had a higher $T_{\text {idle }}$ than those with $\left|t_{\mathrm{err}}\right|=5$ and $10 \mathrm{~s}$. However, the differences between the simulation results with $\left|t_{\mathrm{err}}\right|=5$ and $10 \mathrm{~s}$ were not apparent. Based on the results in Fig. 4, we postulate that $\left(\Delta T_{n i 1}, \Delta T_{n i 2}\right)=(0.5,5)$ days is a well-balanced option between the fuel cost and idle time. Hence, unless explicitly stated, $\Delta T_{n i 1}$ and $\Delta T_{n i 2}$ were set to 0.5 and 5 days, respectively, in the subsequent simulations.

Figure 5 depicts the details of a trajectory correction for $t_{\mathrm{err}}=10 \mathrm{~s}$ and $\left(\Delta T_{n i 1}, \Delta T_{n i 2}\right)=(0,0)$ days. In Fig. 5(a), the dashed and solid lines are the perturbed and correction orbits, respectively. In the correction orbit, 


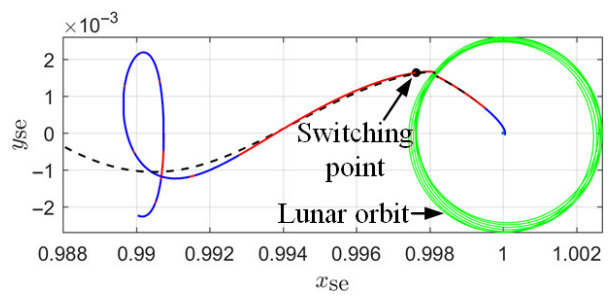

(a) Perturbed and correction orbits

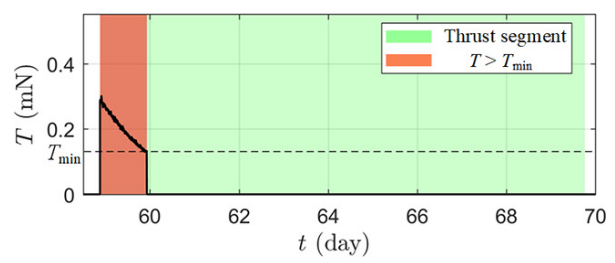

(c) A zoom of $T$ curve
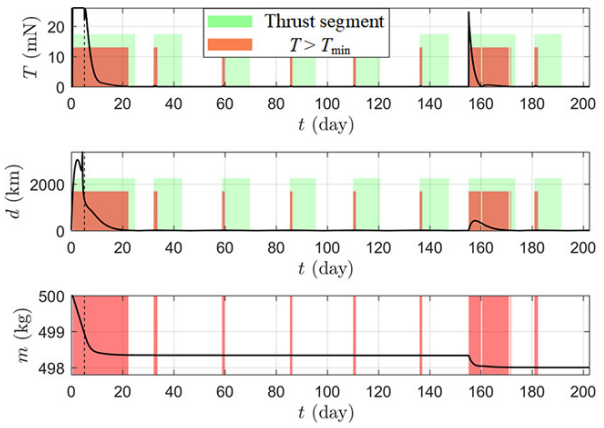

(b) Performance of trajectory correction

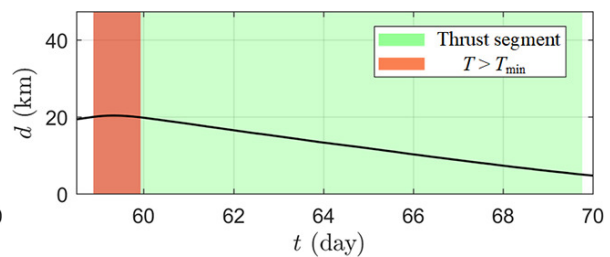

(d) A zoom of $d$ curve

Fig. 5 Trajectory correction for the prograde type 2 orbit without navigation intervals.

blue and red arcs are idle and thrust arcs, respectively. The switching point indicated by a black point is the position at which the $\boldsymbol{Q}$ value changes from $\boldsymbol{Q}_{1}$ to $\boldsymbol{Q}_{2}$. Based on the application scope of $\boldsymbol{Q}_{1}$, it is the point on the lunar flyby transfer whose distance to the EMB is equal to $1.1 a_{\mathrm{m}}\left(1+e_{\mathrm{m}}\right)$. As the figure shows, the perturbed orbit diverged from the reference transfer and passed through the target LPO, but the correction orbit could remain around the reference orbit, and it was inserted into the target LPO. In Fig. 5(b), green and red zones indicate the thrust segment of the dead-band scheme and the actual thrust segment for $T>T_{\min }$, respectively. The dashed vertical lines at $t=5.142$ days in this figure indicate the switching epoch of the $\left\|\boldsymbol{Q}_{1}\right\|$ value. This figure shows that from the beginning of the correction ( $t=0.5$ days $)$ to approximately $t=21$ days, the lowthrust engines continuously operated with a relatively large $T$. During this time, the divergence of the correction orbit from the reference orbit $d$ initially increased and subsequently decreased significantly. Correspondingly, the spacecraft mass significantly decreased owing to fuel consumption during this time. Subsequently, because the divergence of the correction orbit from the reference orbit decreased significantly, several fragmentary short-thrust $\operatorname{arcs}$ with small $T$ values were distributed in the correction orbit 21 days later. When $t$ was approximately 155 days, one more long thrust segment with a larger $T$ appeared because of the insertion maneuver in the reference orbit, but we can observe that this thrust segment was shorter than the first one from $t=0.5-21$ days, and this $d$ was much smaller than the first one. Furthermore, the magnifications of the $T$ and $d$ curves in Fig. 5(b) are shown in Figs. 5(c) and 5(d), respectively. These two figures indicate that the engine limitation and deadband scheme performed their functions in the trajectory correction.

As a comparison, Fig. 6 shows the details of a trajectory correction with $t_{\text {err }}=10 \mathrm{~s}$ and $\left(\Delta T_{n i 1}, \Delta T_{n i 2}\right)=(0.5,5)$ days. In Fig. 6(a), the dashed and solid lines are the perturbed and correction orbits, respectively. In the correction orbit, the blue and red arcs are the idle and thrust arcs, respectively. The switching point of the $\left\|\boldsymbol{Q}_{1}\right\|$ value is indicated by a black point. As the figure shows, this correction orbit is considerably similar to that in Fig. 5(a). Similarly, the green and red zones in Fig. 6(b) are the thrust segment of the dead-band scheme and the actual thrust segment with $T>T_{\min }$, respectively. The dashed vertical lines indicate the switching epoch of the $\left\|\boldsymbol{Q}_{1}\right\|$ value. By comparing Figs. 5(b) and 6(b), we observe that for the two long thrust segments during $t=$ 0.5-21 and 155-171 days, the two trajectory corrections with different navigation intervals were similar. However, from $t=21-155$ days, we observed that the actual thrust segments of the correction orbit with navigation intervals 


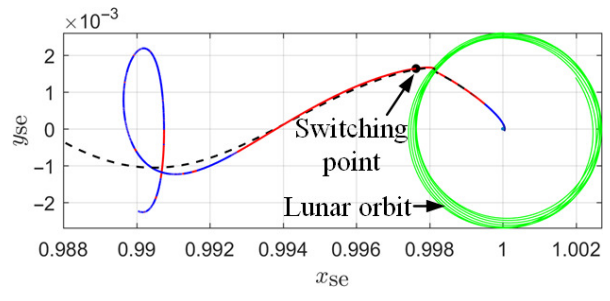

(a) Perturbed and correction orbits

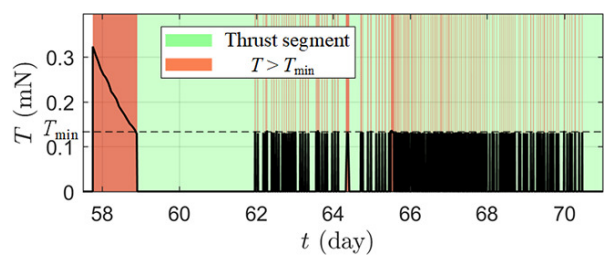

(c) A zoom of $T$ curve
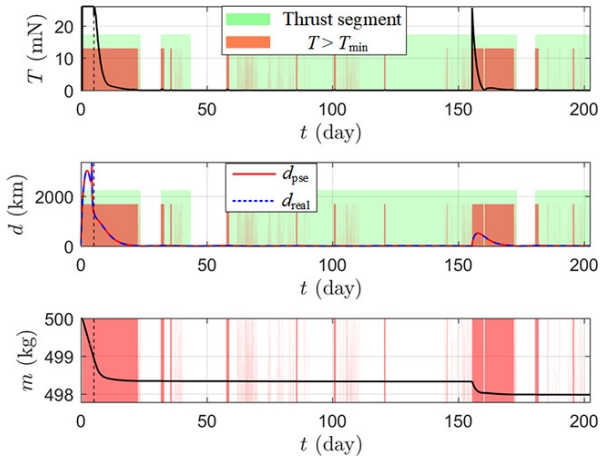

(b) Performance of trajectory correction

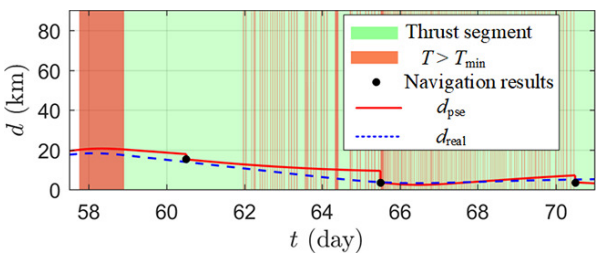

(d) A zoom of $d$ curve

Fig. 6 Trajectory correction for the prograde type 2 orbit with $\left(\Delta T_{n i 1}, \Delta T_{n i 2}\right)=(0.5,5)$ days.

in Fig. 6(b) were much more fragmentary and shorter than those without navigation intervals in Fig. 5(b). This conclusion can be confirmed by the magnifications of the $T$ and $d$ curves in Figs. 6(c) and 6(d). We postulate that the position and velocity deviations could not decrease rapidly to a small range owing to navigation intervals and further resulted in longer thrust segments of the deadband scheme (green zones in Fig. 6(b)). In Fig. 6(d), $d_{\text {real }}$ is the actual position deviation with respect to the reference orbit, and $d_{\mathrm{pse}}$ is the pseudo position deviation obtained using Eq. (12): The curve of $d_{\text {pse }}$ fluctuated around that of $d_{\text {real }}$ in a small range. At the navigation time (see the black points in Fig. 6(d)), the state of spacecraft with navigation errors was obtained by the navigation system. Subsequently, during the navigation interval, the control strategy with practical constraints proposed in the last section was conducted. $d_{\mathrm{pse}}$, obtained from the linear dynamical system, gradually left from $d_{\text {real }}$ over time. However, at the next navigation time, the deflected state of the spacecraft was partially corrected by the navigation system. In addition, the numerical computation indicated that mass losses in the trajectory corrections in Figs. 5 and 6 were considerably similar (approximately $2 \mathrm{~kg}$ ), but the idle time $T_{\text {idle }}$ of the latter was approximately 10 days larger than that of the former, which corresponded with the result shown in Fig. 4.

\subsection{Effects of the dead-band scheme}

In this subsection, we investigate the effects of the deadband scheme on the trajectory correction. As discussed in Section 3, the dead-band scheme is determined by the lower and upper boundaries of the idle segment, $d_{\text {low }}$ and $d_{\text {up }}$, respectively. The previous subsection discusses numerical simulations with the dead-band scheme of $\left(d_{\text {low }}, d_{\text {up }}\right)=(5,20) \mathrm{km}$. In this subsection, two more types of dead-band schemes of $\left(d_{\text {low }}, d_{\mathrm{up}}\right)=$ $(0,0) \mathrm{km}$ and $(10,10) \mathrm{km}$ are shown. Specifically, the trajectory correction with $\left(d_{\text {low }}, d_{\mathrm{up}}\right)=(0,0) \mathrm{km}$ was the one without the dead-band scheme because, based on the definition of the dead-band scheme in Fig. 3, the entire transfer had no idle segments. In the numerical simulations in this subsection, the prograde type 2 transfer in Fig. 1(a) was used as the reference orbit, and practical constraints listed in Table 3 were all considered. The navigation interval was set as $\left(\Delta T_{n i 1}, \Delta T_{n i 2}\right)=(0.5,5)$ days, and the initial launch time error was set as $t_{\text {err }}=10 \mathrm{~s}$.

Figures 7(a) and 7(b) show the performances of two trajectory correction scenarios with $\left(d_{\text {low }}, d_{\text {up }}\right)=(0,0)$ and $(10,10) \mathrm{km}$, respectively. In these figures, green and red zones are the thrust segments of the deadband scheme and the actual thrust segment with $T>$ $T_{\min }$, respectively. The dashed vertical lines indicate 

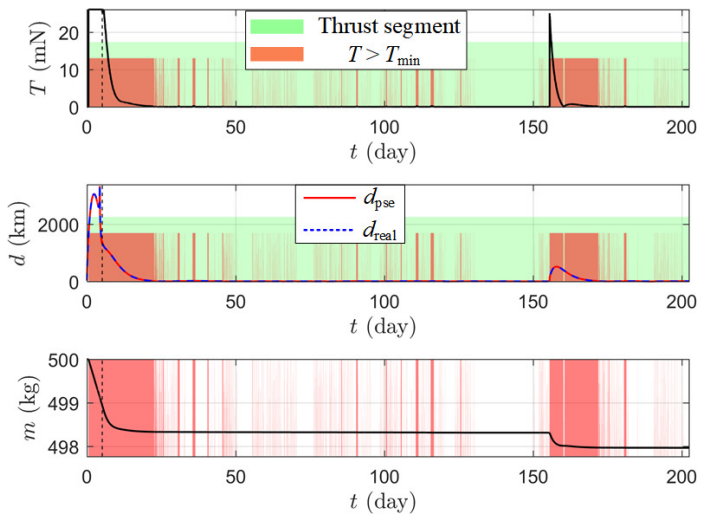

(a) $\left(d_{\text {low }}, d_{\text {up }}\right)=(0,0) \mathrm{km}$
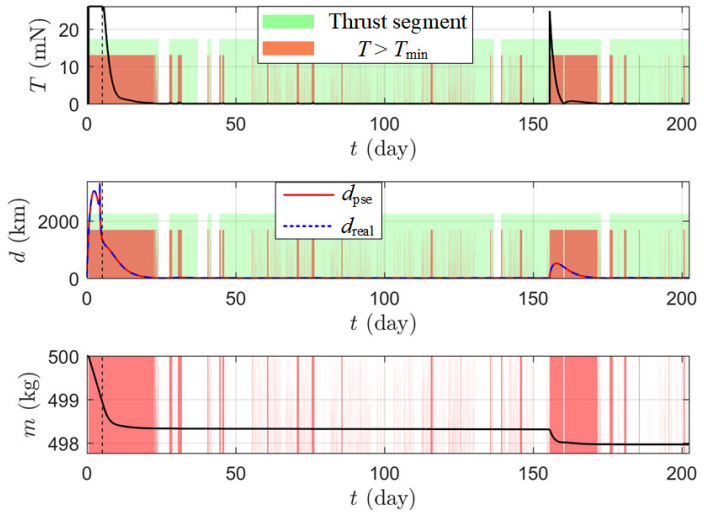

(b) $\left(d_{\text {low }}, d_{\text {up }}\right)=(10,10) \mathrm{km}$

Fig. 7 Performances of two trajectory correction scenario with different dead-band schemes.

the switching epoch of the $\left\|Q_{1}\right\|$ value. $d_{\text {real }}$ is the real position deviation with respect to the reference orbit, and $d_{\text {pse }}$ is the pseudo position deviation obtained by Eq. (12). Comparing with Fig. 6(b), we observe that the $T, d$, and $m$ curves are not noticeably different with different deadband schemes, respectively. The green zones indicate thrust segments of the dead-band scheme. Based on the definition of the dead-band scheme, dead-band schemes with different $\left(d_{\text {low }}, d_{\text {up }}\right)$ had different distributions of thrust segments, and this conclusion can be confirmed by the green regions in Figs. 6 and 7 . We observed that the most significant variation among them was the distribution of green zones. As stated earlier, the trajectory correction without dead-band scheme, i.e., $\left(d_{\text {low }}, d_{\text {up }}\right)=(0,0) \mathrm{km}$, had no idle segments during the entire transfer; therefore, the green zone encompassed the entire transfer. The trajectory correction with the dead-band scheme of $\left(d_{\text {low }}, d_{\text {up }}\right)=(10,10) \mathrm{km}$ had idle segments but its idle segments seemed shorter than those in Fig. 6. In summary, the dead-band scheme on the trajectory correction primarily affected the thrust segment (i.e., green zones in Figs. 6 and 7) rather than the $T, d$, and $m$ curves.

We implemented Monte Carlo simulations to observe the differences in statistics results with different deadband schemes. For each simulation, 100 sample points with practical constraints were selected. As mentioned earlier, the navigation and execution errors are white noise processes and have standard normal distributions with zero means. Table 4 lists the Monte Carlo results of three different dead-band schemes, where Std. is the abbreviation of the standard deviation of the words. The table indicates that the mean values and standard deviations of their mass losses were considerably similar, but the mean idle time $\left(T_{\text {idle }}\right)$ of $\left(d_{\text {low }}, d_{\text {up }}\right)=(5,20) \mathrm{km}$ was larger than those of the other two dead-band schemes. This result can be explained by the observation indicated in Figs. 6 and 7, because the trajectory correction with $\left(d_{\text {low }}, d_{\text {up }}\right)=(5,20) \mathrm{km}$ had the shortest total thrust segment of the dead-band scheme (i.e., the total length of green zones). Therefore, the trajectory correction with $\left(d_{\text {low }}, d_{\text {up }}\right)=(5,20) \mathrm{km}$ had more idle time than those of the other two dead-band schemes.

Table 4 Monte Carlo results of trajectory corrections with different dead-band schemes

\begin{tabular}{lrrr}
\hline$\left(d_{\text {low }}, d_{\text {up }}\right)(\mathrm{km})$ & $(5,20)$ & $(0,0)$ & $(10,10)$ \\
\hline Mean $m_{\text {loss }}(\mathrm{kg})$ & 2.0393 & 2.0391 & 2.0353 \\
Std. $m_{\text {loss }}(\mathrm{kg})$ & 0.0073 & 0.0077 & 0.0072 \\
Mean $T_{\text {idle }}($ day $)$ & 147.8935 & 118.8867 & 120.0013 \\
Std. $T_{\text {idle }}($ day $)$ & 2.4905 & 1.9880 & 2.3296 \\
\hline
\end{tabular}

\subsection{Examples of different lunar flyby transfers}

In this subsection, trajectory corrections for other lunar flyby transfers in Fig. 1 are analyzed and compared. In the numerical simulations of this subsection, the initial launch time error was set as $t_{\text {err }}=10 \mathrm{~s}$, and the deadband scheme of $\left(d_{\text {low }}, d_{\text {up }}\right)=(5,20) \mathrm{km}$ was used in the trajectory corrections. The practical constraints listed in Table 3 were all considered, and the navigation interval was set as $\left(\Delta T_{n i 1}, \Delta T_{n i 2}\right)=(0.5,5)$ days. In addition, control parameters in the backstepping technique, such as $\boldsymbol{S}, \boldsymbol{R}, \boldsymbol{Q}_{1}$, and $\boldsymbol{Q}_{2}$, were identical to the setting for prograde type 2 in Section 3. We adopted the same application scope of $\boldsymbol{Q}_{1}$ as that in Section 3 . 
First, we compare trajectory corrections for the four lunar flyby transfers in Fig. 1 by the Monte Carlo simulations. In the Monte Carlo simulations, for a particular reference orbit, 100 sample points with the initial launch time error and practical constraints were selected. As mentioned earlier, the navigation and maneuver errors listed in Table 3 were white noise processes and had standard normal distributions with zero means. Table 5 shows the Monte Carlo results, where Std. is the abbreviation of the standard deviation of the words. As the table shows, trajectory corrections for prograde types 2 and 4 had similar mean mass losses, which were smaller than those for the retrograde types 2 and 4 . In particular, the perturbed retrograde type 4 transfer had significantly higher fuel consumption or mass loss than the other three lunar flyby transfers. The table indicates that prograde type 2 had the largest mean $\left(T_{\text {idle }}\right)$, and retrograde type 4 had the smallest mean. However, note that the direct comparison of $T_{\text {idle }}$ is meaningless for different lunar flyby transfers because their total flight times (TOFs) differed. The numerical computation indicated that the TOFs of prograde type 2 , prograde type 4 , retrograde type 2 , and retrograde type 4 were 202.3167, 193.9958, 200.7637, and 202.0079 days, respectively. We postulate that $T_{\text {idle }} / \mathrm{TOF}$ can reflect the proportion of idle time during the whole transfer, which is also listed in Table 5 . We can observe that prograde type 2 had the largest proportion of idle time, and retrograde type 4 had the smallest one. The thrust segment of the trajectory correction for the retrograde type 4 occupied approximately half of the transfer time; therefore, it had the largest mass loss. Although prograde type 2 had the largest proportion of idle time, its TOF was larger than the other three cases. Therefore, its mass loss was not the lowest. In addition, based on Eq. (2), the mass loss was determined by the thrust magnitude and thrust time. Hence, even if the prograde type 2 had the shortest thrust time, its total mass loss may not have been the lowest.

Subsequently, we discuss some trajectory correction examples for different lunar flyby transfers in detail. Figure 8 depicts the details of the trajectory correction for the prograde type 4 orbit in Fig. 1(c). In Fig. 8(a), the dashed and solid lines are the perturbed and correction orbits, respectively. In the correction orbit, the blue and red arcs are the idle and thrust arcs, respectively. The switching point of the $\left\|\boldsymbol{Q}_{1}\right\|$ value is indicated by a black point. As the figure shows, the perturbed orbit passed through the target LPO, but the correction orbit could remain around the reference orbit, and it was inserted into the target LPO successively. In Fig. 8(b), the green and red zones are the thrust segment of the

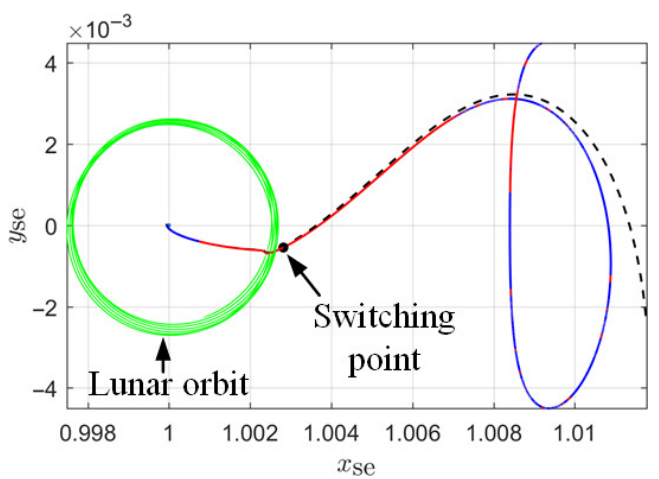

(a) Perturbed and correction orbits
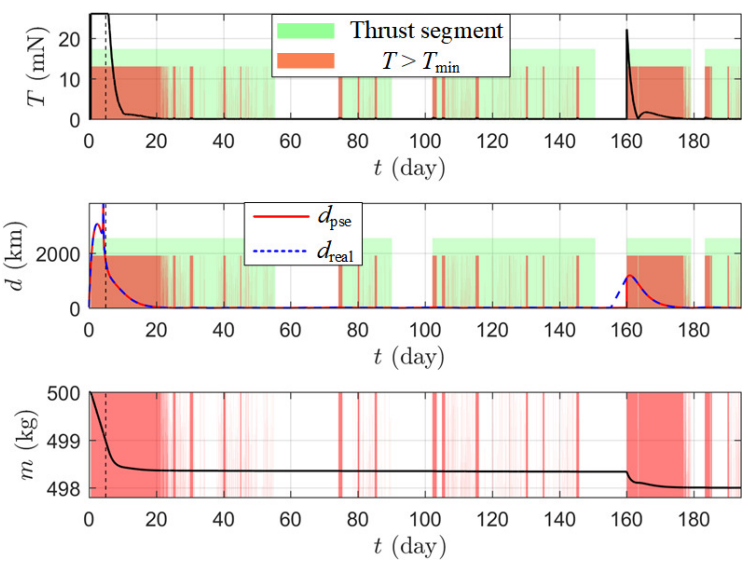

(b) Performance of trajectory correction

Fig. 8 Trajectory correction for the prograde type 4 orbit.

Table 5 Monte Carlo results of trajectory corrections for different lunar flyby transfers

\begin{tabular}{|c|c|c|c|c|}
\hline Transfer type & Prograde 2 & Prograde 4 & Retrograde 2 & Retrograde 4 \\
\hline Mean $m_{\text {loss }}(\mathrm{kg})$ & 2.0393 & 2.0080 & 3.7705 & 11.2331 \\
\hline Std. $m_{\mathrm{loss}}(\mathrm{kg})$ & 0.0073 & 0.0102 & 0.0306 & 0.0853 \\
\hline Mean $T_{\text {idle }}$ (day) & 147.8935 & 138.4160 & 137.9849 & 107.7510 \\
\hline Std. $T_{\text {idle }}$ (day) & 2.4905 & 3.4646 & 2.7536 & 2.5604 \\
\hline Mean $T_{\text {idle }} / \mathrm{TOF}$ & 0.7310 & 0.7135 & 0.6873 & 0.5334 \\
\hline
\end{tabular}


dead-band scheme and the actual thrust segment with $T>T_{\min }$, respectively. The dashed vertical lines indicate the switching epoch of the $\left\|\boldsymbol{Q}_{1}\right\|$ value. $d_{\text {real }}$ is the actual position deviation with respect to the reference orbit, and $d_{\mathrm{pse}}$ is the pseudo position deviation obtained by Eq. (12): We observed that the $T$ curve in Fig. 8(b) was similar to that in Fig. 6(b), but the former had longer green zones than the latter. In addition, we observed that at most times, the curve of $d_{\text {pse }}$ fluctuated around that of $d_{\text {real }}$ in a small range. However, when $t$ was approximately 160 days, $d_{\text {real }}$ was clearly larger than $d_{\text {pse }}$. Numerical computation indicated that the insertion maneuver occurred at $t=155.23$ days, and the latest navigation epoch occurred at $t=155$ days. Because $d_{\text {pse }}$ was calculated over almost 5 days according to the state before the insertion maneuver, its curve diverged from $d_{\text {real }}$ until the next navigation epoch $t=160$ days.

Figure 9 shows the detail of a trajectory correction for the retrograde type 2 orbit in Fig. 1(b). In Fig. 9(a), the dashed and solid lines denote the perturbed and correction orbits, respectively. In the correction orbit, the

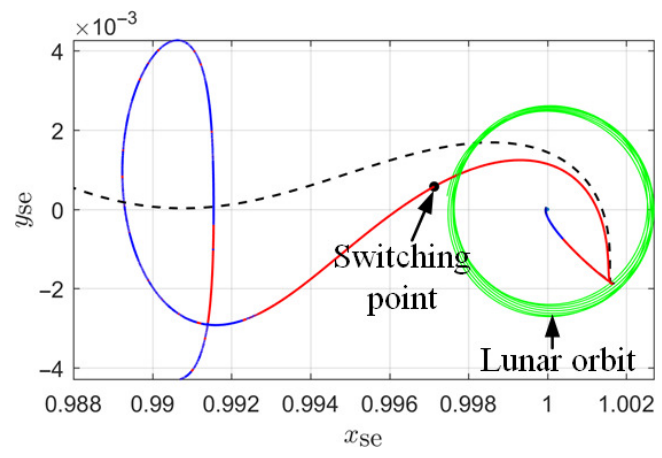

(a) Perturbed and correction orbits
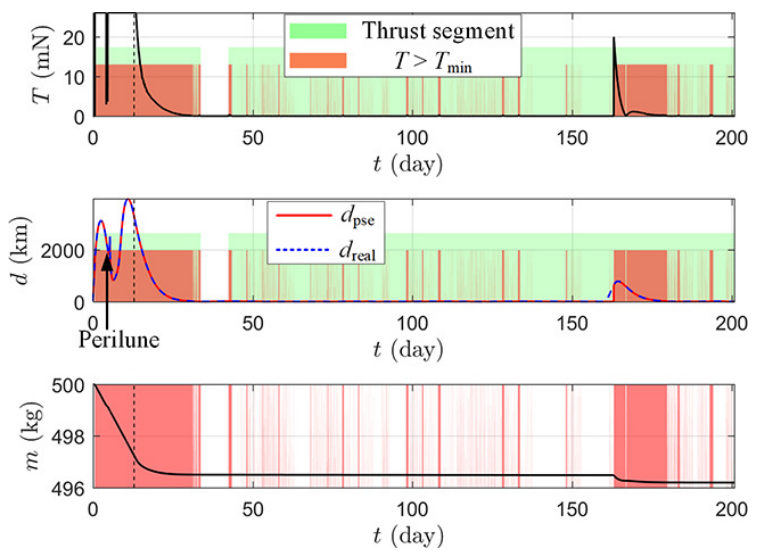

(b) Performance of trajectory correction

Fig. 9 Trajectory correction for the retrograde type 2 orbit. blue and red arcs are idle and thrust arcs, respectively. The switching point of the $\left\|\boldsymbol{Q}_{1}\right\|$ value is represented by a black point. The correction orbit could remain around the reference orbit and was inserted into the target LPO successively. Compared with trajectory corrections for prograde lunar flyby orbits in Figs. 6 and 8, we observe that both $T$ and $d$ curves of the retrograde type 2 orbit are significantly different. The trajectory correction for the retrograde type 2 orbit had a longer thrust segment with $T=T_{\max }$ than the above two prograde lunar flyby orbits because its $d$ curve could not rapidly decrease to a small value after the lunar flyby similar to the prograde lunar flyby orbits. As Fig. 9(b) shows, the $d$ curve has two peaks before the switching point. Numerical computation indicated that the first peak occurred before the lunar flyby, and the second one appears before the spacecraft passed through the lunar orbit. We consider that this phenomenon can be explained by the special shape of retrograde lunar flyby transfers. As Fig. 9(a) shows, the retrograde lunar flyby orbit experienced a lunar flyby and an Earth flyby, successively, before the switching point. The Earth flyby, similar to the lunar flyby, could also result in a significant divergence of the perturbed orbit from the reference transfers. Therefore, the $d$ curve has two peaks before the switching point. For the retrograde type 2 orbit, a longer flight time in the lunar orbit resulted in a second increase in $d$ before the switching point. In addition, we observed that when the insertion maneuver occurred at $t=160.7$ days, the divergence of $d_{\text {pse }}$ from $d_{\text {real }}$ was smaller than that in Fig. 8, because the next navigation epoch at $t=163$ days was close to the insertion maneuver point.

Figure 10 depicts the details of a trajectory correction for the retrograde type 4 orbit in Fig. 1(d). In Fig. 10(a), the dashed and solid lines are the perturbed and correction orbits, respectively. In the correction orbit, the blue and red arcs are the idle and thrust arcs, respectively. The switching point of the $\left\|Q_{1}\right\|$ value is represented by a black point. The correction orbit could remain around the reference orbit and was inserted into the target LPO successively. Compared with trajectory corrections for the other three lunar flyby orbits, we observed that the trajectory correction for the retrograde type 4 orbit had a much longer thrust segment with $T=T_{\max }$ (approximately 40 days) because its $d$ curve could not rapidly decrease to a small value after the lunar flyby, and it even increased to approximately $3.2 \times 10^{4}$ 


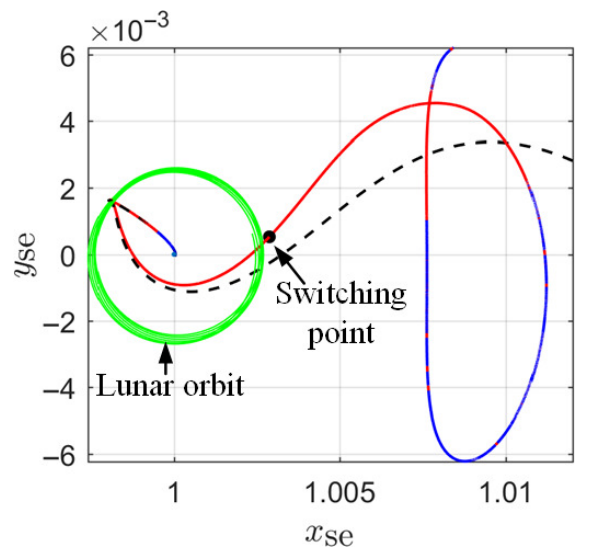

(a) Perturbed and correction orbits
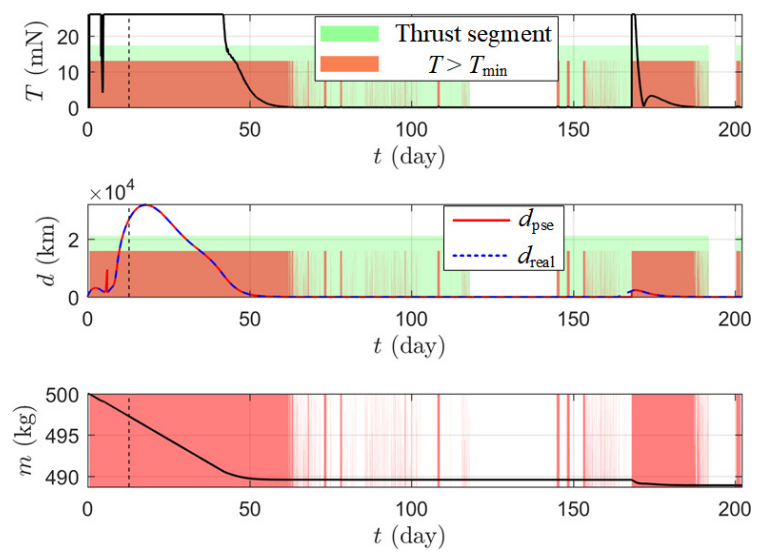

(b) Performance of trajectory correction

Fig. 10 Trajectory correction for the retrograde type 4 orbit.

$\mathrm{km}$ after the switching point. Moreover, the numerical computation indicated that a long flight time in the lunar orbit resulted in an increase in $d$ before and after the switching point. In addition, numerical results indicated that, in the navigation interval $t \in[163,168]$ days, the insertion maneuver occurred at $t=163.6$ days and the divergence of $d_{\text {pse }}$ from $d_{\text {real }}$ exceeded $2000 \mathrm{~km}$, which was much larger than the other three lunar flyby orbits.

\section{Conclusions}

In this paper, trajectory corrections for lunar flyby transfers to Sun-Earth/Moon LPOs with continuous thrusts were investigated using the ephemeris model. Although many studies on trajectory corrections for transfers to LPOs have been conducted, trajectory corrections for lunar flyby transfers are insufficient and significantly different from those without lunar flyby because of their special geometrical and dynamical structures. Lunar flyby transfer is a high-yielding but high-risk design method; therefore, its TCM strategy should be specifically designed. In this paper, we first introduce the backstepping technique into the trajectory correction for lunar flyby transfers to LPOs with practical constraints. Numerical simulations indicated that our trajectory correction method is feasible and efficient for problems with different practical constraints. In contrast to the previous reference on this problem, the propellant system in this study was the continuous thrust rather than the impulsive thrust. In addition, compared with a previous study, more practical constraints in the trajectory correction were considered in this study, such as the dead-band scheme, navigation intervals, execution error, and the limitation of the thrust engine. Therefore, the control strategy proposed herein is more realistic than that in the previous study. A control strategy based on the backstepping technique with a dead-band scheme was proposed using the ephemeris model. Since the perturbed transfers significantly diverge from the reference transfers after the spacecraft passes by the Moon, we postulated that using two sets of the parameters $\boldsymbol{S}, \boldsymbol{Q}$, and $\boldsymbol{R}$ is necessary in two portions before and after the lunar flyby. Based on numerical analysis and computation, we selected a larger $\boldsymbol{Q}$ in the portion of the prior-lunar flyby to increase the convergence rate of the perturbed orbit to the reference orbit, although it could result in a larger thrust acceleration. For the post-lunar flyby, we adopted a smaller $\boldsymbol{Q}$ to reduce the thrust acceleration. According to the distance of the spacecraft to the EarthMoon barycenter, the application scope of the larger $\boldsymbol{Q}$ is defined.

Using a prograde type 2 orbit as the reference orbit, the effects of navigation intervals on trajectory corrections are discussed using the results of numerical simulations. Numerical computation showed that for a particular launch time error, trajectory corrections with different navigation intervals have almost similar mass losses, but the trajectory correction with longer navigation intervals has a longer idle time. Numerical results indicated that $\left(\Delta T_{n i 1}, \Delta T_{n i 2}\right)=(0.5,5)$ days is a wellbalanced option between fuel consumption and idle time. The detailed analysis showed that the actual thrust segments of the correction orbit with navigation intervals are much more fragmentary and shorter than those without navigation intervals because the former has longer thrust segments of the dead-band scheme. Subsequently, we implemented numerical simulations to 
observe the differences in trajectory corrections with different dead-band schemes. A dead-band scheme can avoid thrusters operating continuously for long-term maneuvers. Numerical computation showed that the effects of the dead-band scheme on the trajectory correction primarily appeared in the thrust segments of the dead-band scheme. Monte Carlo simulations indicated that mass losses with different dead-band schemes were significantly similar. Finally, trajectory corrections for different lunar flyby transfers are shown and compared. Monte Carlo simulations showed that trajectory corrections for prograde types 2 and 4 had similar mean mass losses, which were smaller than those for retrograde types 2 and 4 . In particular, the perturbed retrograde type 4 transfer had significantly higher fuel consumption or mass loss than the other three lunar flyby transfers. We observed that prograde type 2 had the largest proportion of idle time, and retrograde type 4 had the smallest one. Compared with the prograde lunar flyby orbits, the retrograde lunar flyby orbits had a longer flight time in the lunar orbit, which resulted in larger position deviations with respect to the reference orbits and higher fuel consumption.

\section{Acknowledgements}

This work was supported by the Canada Research Chair Program under Grant No. 950-230883.

\section{Declaration of competing interest}

The authors have no competing interests to declare that are relevant to the content of this article.

\section{References}

[1] Farquhar, R. W. The flight of ISEE-3/ICE: Origins, mission history, and a legacy. The Journal of the Astronautical Sciences, 2001, 49(1): 23-73.

[2] Ogilvie, K. W., Desch, M. D. The WIND spacecraft and its early scientific results. Advances in Space Research, 1997, 20(4-5): 559-568.

[3] Domingo, V., Fleck, B., Poland, A. I. The SOHO mission: An overview. Solar Physics, 1995, 162(1-2): 1-37.

[4] Bennett, C. L., Bay, M., Halpern, M., Hinshaw, G., Jackson, C., Jarosik, N., Kogut, A., Limon, M., Meyer, S. S., Page, L. et al. The microwave anisotropy probe mission. The Astrophysical Journal Letters, 2003, 583(1): $1-23$.

[5] Prusti, T., de Bruijne, J. H. J., Brown, A. G. A., Vallenari, A., Babusiaux, C., Bailer-Jones, C. A. L.,
Bastian, U., Biermann, M., Evans, D. W., Eyer, L. et al. The Gaia mission. Astronomy \& Astrophysics, 2016, 595: A1.

[6] Wu, W. R., Liu, Y., Liu, L., Zhou, J. L., Tang, G. S., Chen, Y. Z. Pre-LOI trajectory maneuvers of the CHANG'E-2 libration point mission. Science China Information Sciences, 2012, 55(6): 1249-1258.

[7] Gómez, G., Jorba, A., Masdemont, J., Simó, C. A dynamical systems approach for the analysis of the SOHO mission. In: Proceedings of the 3rd International Symposium on Spacecraft Flight Dynamics, 1991: 449454.

[8] Sukhanov, A., Prado, A. F. B. A. Lambert problem solution in the hill model of motion. Celestial Mechanics and Dynamical Astronomy, 2004, 90(3-4): 331-354.

[9] Renk, F., Landgraf, M. Sun-Earth Libration point transfer options with intermediate HEO. Acta Astronautica, 2012, 74: 1-19.

[10] Peng, H., Bai, X. L., Masdemont, J. J., Gómez, G., Xu, S. J. Libration transfer design using patched elliptic threebody models and graphics processing units. Journal of Guidance, Control, and Dynamics, 2017, 40(12): 31553166.

[11] Qi, Y., Xu, S. J., Qi, R. Transfer from Earth to libration point orbit using lunar gravity assist. Acta Astronautica, 2017, 133: 145-157.

[12] Farquhar, R. W., Muhonen, D. P., Newman, C. R., Heubergerg, H. S. Trajectories and orbital maneuvers for the first libration-point satellite. Journal of Guidance and Control, 1980, 3(6): 549-554.

[13] Serban, R., Koon, W. S., Lo, M. W., Marsden, J. E., Petzold, L. R., Ross, S. D., Wilson, R. S. Halo orbit mission correction maneuvers using optimal control. Automatica, 2002, 38(4): 571-583.

[14] Gómez, G., Marcote, M., Masdemont, J. J. Trajectory correction manoeuvres in the transfer to libration point orbits. Acta Astronautica, 2005, 56(7): 652-669.

[15] Xu, M., Xu, S. J. Trajectory and correction maneuver during the transfer from earth to halo orbit. Chinese Journal of Aeronautics, 2008, 21(3): 200-206.

[16] Salmani, M., Büskens, C. Real-time control of optimal low-thrust transfer to the Sun-Earth L1 halo orbit in the bicircular four-body problem. Acta Astronautica, 2011, 69(9-10): 882-891.

[17] Peng, H. J., Gao, Q., Wu, Z. G., Zhong, W. X. Optimal guidance based on receding horizon control for low-thrust transfer to libration point orbits. Advances in Space Research, 2013, 51(11): 2093-2111.

[18] Qi, Y., de Ruiter, A. Study of correction maneuver for lunar flyby transfers in the real ephemeris. Journal of 
Guidance, Control, and Dynamics, 2018, 41(10): 21122132 .

[19] Nazari, M., Anthony, W. M., Butcher, E. Continuous thrust stationkeeping in Earth-Moon L1 halo orbits based on LQR control and floquet theory. In: Proceedings of AIAA/AAS Astrodynamics Specialist Conference, 2014: AIAA 2014-4140.

[20] Qi, Y., de Ruiter, A. Station-keeping strategy for real translunar libration point orbits using continuous thrust. Aerospace Science and Technology, 2019, 94: 105376.

[21] Jiang, Y., Hu, Q. L., Ma, G. F. Adaptive backstepping fault-tolerant control for flexible spacecraft with unknown bounded disturbances and actuator failures. ISA Transactions, 2010, 49(1): 57-69.

[22] Folkner, W. M., Williams, J. G., Boggs, D. H., Park, R. S., Kuchynka, P. The planetary and lunar ephemerides DE430 and DE431. The Interplanetary Network Progress Report, 2014, (196): 1-81.

[23] Deshmukh, V. S., Sinha, S. C. Control of dynamic systems with time-periodic coefficients via the Lyapunovfloquet transformation and backstepping technique. Journal of Vibration and Control, 2004, 10(10): 15171533.

[24] Qi, Y., Xu, S. J. Study of lunar gravity assist orbits in the restricted four-body problem. Celestial Mechanics and Dynamical Astronomy, 2016, 125(3): 333-361.

[25] Sweetser, T. H., Broschart, S. B., Angelopoulos, V., Whiffen, G. J., Folta, D. C., Chung, M. K., Hatch, S. J., Woodard, M. A. ARTEMIS mission design. Space Science Reviews, 2011, 165(1-4): 27-57.

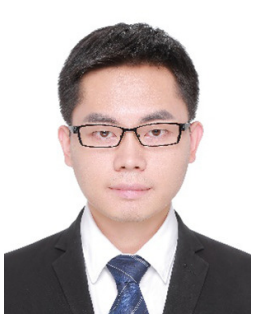

Yi Qi received his Ph.D. degree in aeronautical and astronautical science and technology from Beihang University, China, in 2017. After three years as a postdoctoral researcher in Ryerson University, he joined Beijing Institute of Technology as an associate professor in 2020. His research area includes orbital dynamics and control for deep space exploration. E-mail: lushenqiyi@gmail.com.

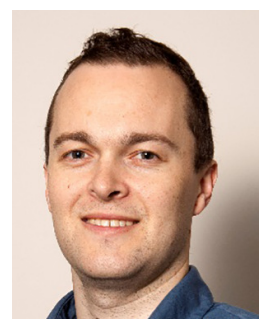

Anton de Ruiter received his B.E. degree in mechanical engineering from the University of Canterbury in 1999, and M.A.Sc. and Ph.D. degrees in aerospace engineering from the University of Toronto in 2001 and 2005, respectively. Between 2006 and 2008 he was a visiting research fellow at the Canadian Space Agency in Montreal, and an assistant professor in the Department of Mechanical and Aerospace Engineering at Carleton University from 2009 to 2012 . He is currently an associate professor and Canada research chair (Tier 2) in the Department of Aerospace Engineering at Ryerson University in Toronto, Canada. His research interests are in the area of guidance, navigation, and control of aerospace systems. E-mail: aderuiter@ryerson.ca.

Open Access This article is licensed under a Creative Commons Attribution 4.0 International License, which permits use, sharing, adaptation, distribution and reproduction in any medium or format, as long as you give appropriate credit to the original author(s) and the source, provide a link to the Creative Commons licence, and indicate if changes were made.

The images or other third party material in this article are included in the article's Creative Commons licence, unless indicated otherwise in a credit line to the material. If material is not included in the article's Creative Commons licence and your intended use is not permitted by statutory regulation or exceeds the permitted use, you will need to obtain permission directly from the copyright holder.

To view a copy of this licence, visit http://creativecoorg/li censes/by $/ 4.0 /$. 